\title{
THERE IS NO STRICTLY SINGULAR CENTRALIZER ON $L_{p}$
}

\author{
FÉLIX CABELLO SÁNCHEZ
}

(Communicated by Thomas Schlumprecht)

\begin{abstract}
We prove that if $\Phi$ is a centralizer on $L_{p}$, where $0<p<\infty$, then there is a copy of $\ell_{2}$ inside $L_{p}$ where $\Phi$ is bounded. If $\Phi$ is symmetric, then it is also bounded on a copy of $\ell_{q}$, provided $0<p<q<2$. This shows that for a wide class of exact sequences $0 \rightarrow L_{p} \rightarrow Z \rightarrow L_{p} \rightarrow 0$ the quotient map is not strictly singular, which generalizes a recent result of Jesús Suárez.
\end{abstract}

\section{INTRODUCTION}

An operator acting between Banach or quasi-Banach spaces is said to be strictly singular if it is not an isomorphism on any infinite dimensional subspace of its domain.

Exact sequences of Banach or quasi-Banach spaces $0 \rightarrow Y \rightarrow Z \rightarrow X \rightarrow 0$ in which the quotient map $\pi: Z \rightarrow X$ is strictly singular has spurred moderate interest since the early studies on the 'three space problem'. Let us call them 'strictly singular sequences'. In some sense, if one has a strictly singular sequence in which the spaces $X$ and $Y$ are 'nice', the middle space $Z$ must be 'exotic'.

Amongst the most striking examples of this phenomenon one finds that for each $p \in(0, \infty)$ there is a strictly singular sequence

$$
0 \longrightarrow \ell_{p} \longrightarrow Z_{p} \longrightarrow \ell_{p} \longrightarrow 0 \text {. }
$$

These were constructed by Kalton and Peck in [9]; see also [3].

More often than not the construction of strictly singular sequences is achieved by means of a quasilinear map from $X$ to $Y$, and this is certainly the case for the Kalton-Peck sequences, whose associated quasilinear maps are centralizers (a special type of quasilinear map; see Section 1.2). There is a function space analogue of (1),

$$
0 \longrightarrow L_{p} \longrightarrow F_{p} \longrightarrow L_{p} \longrightarrow 0,
$$

whose associated quasilinear map is the 'classical' centralizer

$$
\Omega(f)=f \log \left(\frac{|f|}{\|f\|}\right) .
$$

Received by the editors January 31, 2012 and, in revised form, April 16, 2012 and April 19, 2012 .

2010 Mathematics Subject Classification. Primary 47B10, 46M18, 46A16.

Key words and phrases. Strictly singular operator, quasilinear centralizer, Rademacher functions, $q$-stable random variable.

This work was supported in part by MTM2010-20190-C02-01 and Junta de Extremadura CR10113 "IV Plan Regional I+D+i, Ayudas a Grupos de Investigación". 
The space $Z F_{p}$ was introduced in [5], although it arises quite naturally in interpolation theory; see [10, Section 3D].

Very recently Jesús Suárez proved the following remarkable results on the behaviour of $\Omega$ on $L_{p}$ :

(a) For every $0<p<\infty$, there is a copy of $\ell_{2}$ in $L_{p}$ where the restriction of $\Omega$ is bounded.

(b) If $0<p<q<2$, then $\Omega$ is bounded on a copy of $\ell_{q}$ inside $L_{p}$.

See [12, Propositions 3.1 and 4.1]. Roughly this means that the sequence (2) is not strictly singular because the quotient map is invertible on an isomorphic copy of $\ell_{2}$ (or $\ell_{q}$ ) inside $L_{p}$.

The purpose of this short note is to prove that (a) holds for all centralizers and (b) holds (at least) for symmetric centralizers. Our approach is based on a result by Kalton that describes centralizers as differentials of interpolation scales of Köthe function spaces from [7. We also use results from [6] and a recent result of the author on the behaviour of centralizers acting between two different Lebesgue spaces [2].

1.1. Function spaces. Let $L_{0}$ denote the space of all real or complex measurable functions on the unit interval $\mathbb{I}$, where we identify two functions if they agree almost everywhere with respect to Lebesgue measure. A function space $X$ is a linear subspace of $L_{0}$, together with a quasinorm $\|\cdot\|$ having the following properties:

- The unit ball $B_{X}=\{f \in X:\|f\| \leq 1\}$ is closed in $L_{0}$ for the topology of convergence in measure.

- If $f \in X, g \in L_{0}$ and $|g| \leq|f|$, then $g \in X$ and $\|g\| \leq\|f\|$.

Important examples of function spaces are the spaces $L_{p}$ for $0<p \leq \infty$.

Given a function space $X$ and $A \subset \mathbb{I}$ we write $X(A)$ for the space of those functions in $X$ vanishing outside $A$.

We consider Köthe function spaces in the sense of [7. Thus they are Banach function spaces whose norm satisfies the inequalities $\|h x\|_{1} \leq\|x\|_{X} \leq\|k x\|_{\infty}$ for some everywhere positive functions $h, k$ and for every $x \in X$.

1.2. Centralizers and extensions. Let $X$ and $Y$ be function spaces. A centralizer from $X$ to $Y$ is a homogeneous mapping $\Phi: X \rightarrow L_{0}$ satisfying the following condition: there is a constant $C$ such that, for every $a \in L_{\infty}$ and for every $f \in X$, the difference $\Phi(a f)-a \Phi(f)$ belongs to $Y$ and

$$
\|\Phi(a f)-a \Phi(f)\|_{Y} \leq C\|a\|_{\infty}\|f\|_{X} .
$$

When $Y=X$ we say that $\Phi$ is a centralizer on $X$.

Although we will not use it, we remark that every centralizer is quasilinear; that is, there is a constant $Q$ such that for every $f, g \in X$ the difference $\Phi(f+g)-\Phi f-\Phi g$ falls in $Y$ and one has $\|\Phi(f+g)-\Phi f-\Phi g\|_{Y} \leq Q\left(\|f\|_{X}+\|g\|_{X}\right)$.

A centralizer from $X$ to $Y$ gives rise to an exact sequence

$$
0 \longrightarrow Y \stackrel{\imath}{\longrightarrow} Y \oplus_{\Phi} X \stackrel{\pi}{\longrightarrow} X \longrightarrow 0
$$

as follows:

- The middle space is $Y \oplus_{\Phi} X=\left\{(g, f) \in L_{0} \times X: g-\Phi(f) \in Y\right\}$ with the quasinorm given by $\|(g, f)\|_{\Phi}=\|g-\Phi f\|_{Y}+\|f\|_{X}$.

- $\imath(g)=(g, 0)$ and $\pi(g, f)=f$. 
Actually only quasilinearity of $\Phi$ is required here.

We say that two centralizers $\Phi$ and $\Psi$ are equivalent, and we write $\Phi \approx \Psi$ if the difference takes values in $Y$ and is bounded in the sense that $\|\Phi(f)-\Psi(f)\|_{Y} \leq$ $B\|f\|_{X}$ for some $B$ and every $f \in X$.

Let $U$ be a subspace of $X$ and suppose $\Phi$ is bounded on $U$ in the sense that $\Phi$ maps $U$ into $Y$ (not $L_{0}$ ) and $\|\Phi(u)\|_{Y} \leq B\|u\|_{X}$ for some constant $B$ and every $u \in U$. Then the map $s: U \rightarrow Y \oplus_{\Phi} X$ defined by $s(u)=(0, u)$ is a bounded linear operator and $\pi \circ s=I_{U}$. Thus $\pi$ cannot be strictly singular if $\Phi$ is bounded on some infinite dimensional subspace of $X$.

Important examples of centralizers are the following (see [6], Section 3 and especially Theorem 3.1). Let $\varphi: \mathbb{R}^{2} \rightarrow \mathbb{R}$ be a Lipschitz function vanishing at the origin. Then the map $L_{p} \rightarrow L_{0}$ given by

$$
f \longmapsto f \varphi\left(\log \frac{|f|}{\|f\|_{p}}, \log \frac{\left|r_{f}\right|}{\|f\|_{p}}\right)
$$

is a (real, symmetric) centralizer on $L_{p}$. Here $r_{g}$ is the so-called rank-function of $g \in L_{0}$ defined by

$$
r_{g}(t)=\lambda\left\{s \in \mathbb{R}^{+}:|g(s)|>|g(t)| \text { or } s \leq t \text { and }|g(s)|=|g(t)|\right\},
$$

which arises in real interpolation.

1.3. Real centralizers. Let $X$ be a complex function space and let $X^{\mathbb{R}}=\Re(X)$ be a corresponding real function space. A centralizer on $X$ is said to be real if it sends real functions into real functions. Clearly, every real centralizer on $X$ induces a centralizer on $X^{\mathbb{R}}$ by restriction. On the other hand each centralizer $\Phi$ on $X^{\mathbb{R}}$ extends to a real centralizer on $X$ by the formula $\Phi^{\mathbb{C}} f=\Phi(u)+i \Phi(v)$, where $u=\Re f$ and $v=\Im f$. These processes are each inverse of the other, up to equivalence.

Moreover, if $\Phi$ is any centralizer on $X$, there are real centralizers $\Phi_{1}$ and $\Phi_{2}$ such that $\Phi \approx \Phi_{1}+i \Phi_{2} ;$ see [7, Lemma 7.1].

\section{Results}

Let $A$ be a Borel subset of $\mathbb{I}$. A Rademacher sequence in $A$ is a sequence $\left(r_{n}\right)$ in $L_{0}(A)$ such that $\lambda\left(\left\{t \in A: r_{n}(t)=1\right\}\right)=\lambda\left(\left\{t \in A: r_{n}(t)=-1\right\}\right)=\frac{1}{2} \lambda(A)$ for all $n$ and $\mathbb{E}\left[r_{n} r_{m} \mid A\right]=0$ for $n \neq m$.

Khintchine's inequality states that if $\left(r_{n}\right)$ is a Rademacher sequence and $\left(t_{n}\right)$ is in $\ell_{2}$, then $f=\sum_{n} t_{n} r_{n}$ belongs to $L_{s}$ for every $s \in(0, \infty)$ and, moreover, there is a constant $M$ depending only on $s$ and $\lambda(A)$ such that

$$
M^{-1}\left\|\left(t_{n}\right)\right\|_{\ell_{2}} \leq\|f\|_{s} \leq M\left\|\left(t_{n}\right)\right\|_{\ell_{2}} .
$$

Thus a Rademacher sequence spans a subspace isomorphic to $\ell_{2}$ in $L_{s}$ for any $s \in(0, \infty)$.

Our first result is based on certain ideas from complex interpolation. Let us indicate the minimal background one needs to understand the proof.

Let $X_{0}$ and $X_{1}$ be (complex) Köthe function spaces on the unit interval. Consider the closed strip $\mathbb{S}=\{z \in \mathbb{C}: 0 \leq \Re(z) \leq 1\}$ and let $\mathcal{F}\left(X_{0}, X_{1}\right)$ denote the space of bounded, continuous functions $F: \mathbb{S} \rightarrow X_{0}+X_{1}$ having the following properties:

- $F$ is analytic on the interior of $\mathbb{S}$.

- $F(k+i t) \in X_{k}$ for each $k=0,1$ and all $t \in \mathbb{R}$.

- For $k=0,1$, the map $t \in \mathbb{R} \mapsto F(k+i t) \in X_{k}$ is bounded and continuous. 
Then $\mathcal{F}=\mathcal{F}\left(X_{0}, X_{1}\right)$ is a Banach space under the norm

$$
\|F\|_{\mathcal{F}}=\sup \left\{\|F(k+i t)\|_{X_{k}}: t \in \mathbb{R}, k=0,1\right\} .
$$

For $\theta \in[0,1]$ we define the interpolation space

$$
X_{\theta}=\left[X_{0}, X_{1}\right]_{\theta}=\left\{f \in L_{0}: f=F(\theta) \text { for some } F \in \mathcal{F}\right\}
$$

with the (quotient) norm $\|f\|_{X_{\theta}}=\inf \left\{\|F\|_{\mathcal{F}}: f=F(\theta)\right\}$.

The equation $\left[X_{0}, X_{1}\right]_{\theta}=X$ induces a 'derivation' on $X$ as follows. We fix a small $\epsilon>0$ and for each $f \in X$ we choose $F \in \mathcal{F}\left(X_{0}, X_{1}\right)$ such that $F(\theta)=f$, with $\|F\|_{\mathcal{F}} \leq(1+\epsilon)\|f\|_{X}$. Then we put $\Omega(f)=F^{\prime}(\theta)$. The map $\Omega: X \rightarrow L_{0}$ is a centralizer on $X$, and two centralizers obtained with different choices of $F$ are equivalent.

An important result of Kalton [7, Theorem 7.6] states that if $\Phi$ is a real centralizer on $L_{p}$, with $p>1$, then there is a constant $c>0$ and a couple of Köthe functions spaces such that $L_{p}=\left[X_{0}, X_{1}\right]_{\theta=1 / 2}$ with equivalent norms, in the sense that both spaces contain the same functions and there is $M$ such that

$$
M^{-1}\|f\|_{p} \leq \inf _{f=F\left(\frac{1}{2}\right)}\|F\|_{\mathcal{F}} \leq M\|f\|_{p}
$$

for all $f \in L_{p}$, and $\Phi \approx c \Omega$, where $\Omega$ is the corresponding derivation on $X_{1 / 2}=L_{p}$.

Proposition 1. Let $\Phi$ be a centralizer on $L_{p}$, where $0<p<\infty$. Then for each $\delta>0$ there is a set $B \subset \mathbb{I}$ with $\lambda(B) \geq 1-\delta$ such that, for each $A \subset B$ of positive measure, $\Phi$ is bounded on the closed subspace spanned by any Rademacher sequence in $A$. In particular, the sequence $0 \rightarrow L_{p} \rightarrow L_{p} \oplus_{\Phi} L_{p} \rightarrow L_{p} \rightarrow 0$ is not strictly singular.

Proof. It should be clear from the remarks in Section 1.3 that it suffices to prove the proposition assuming that $\Phi$ is a real centralizer on the complex $L_{p}$.

First suppose $p>1$. Then by the result of Kalton quoted above, we know that there are a couple of Köthe spaces $\left(X_{0}, X_{1}\right)$ and $c>0$ such that $L_{p}=\left[X_{0}, X_{1}\right]_{1 / 2}$ and $\Phi \approx c \Omega$.

Let us take a look at $\Omega$. First, by iteration, we have $L_{p}=\left[X_{1 / 4}, X_{3 / 4}\right]_{1 / 2}$ where $X_{k / 4}=\left[X_{0}, X_{1}\right]_{k / 4}$ for $k=1,3$ and both $X_{1 / 4}$ and $X_{3 / 4}$ are super-reflexive by [8. Theorem 5.8]. On the other hand, if $F \in \mathcal{F}\left(X_{0}, X_{1}\right)$, then the function $G$ defined by $G(z)=F\left(\frac{1}{2}\left(z+\frac{1}{2}\right)\right)$ belongs to $\mathcal{F}\left(X_{1 / 4}, X_{3 / 4}\right)$ and one has $\|G\|_{\mathcal{F}} \leq$ $\|F\|_{\mathcal{F}}, G\left(\frac{1}{2}\right)=F\left(\frac{1}{2}\right)$ and $G^{\prime}\left(\frac{1}{2}\right)=\frac{1}{2} F\left(\frac{1}{2}\right)$.

Thus replacing the couple $\left(X_{0}, X_{1}\right)$ by $\left(X_{1 / 4}, X_{3 / 4}\right)$ preserves the induced centralizer, up to a constant factor, and so we may assume $X_{0}$ and $X_{1}$ are superreflexive Köthe spaces.

Now, for $i=0,1$, take everywhere positive functions $h_{i}$ and $k_{i}$ so that $\left\|h_{i} f\right\|_{1} \leq$ $\|f\|_{X_{i}} \leq\left\|k_{i} f\right\|_{\infty}$ for all $f \in X_{i}$ and observe that for fixed $\delta>0$ there is $M$ large enough and a subset $B \subset \mathbb{I}$ with $\lambda(B)>1-\delta$ where $k_{i} \leq M$ and $h_{i} \geq 1 / M$ for $i=0,1$.

It follows that $L_{\infty}(B) \subset X_{i}(B) \subset L_{1}(B)$, with continuous inclusions, and since $X_{i}$ is super-reflexive it is also $s_{i}$-concave for some finite $s_{i}$, and so we have a continuous inclusion $L_{s_{i}}(B) \subset X_{i}(B)$ (see 4, p. 14]). Now taking $s=\max s_{i}$ we conclude that $L_{s}(B)$ embeds continuously into $X_{i}$, and so there is a constant $M$ such that $\|f\|_{X_{i}} \leq M\|f\|_{s}$ for every $f \in L_{s}(B)$ and $i=0,1$.

Now, let $\left(r_{n}\right)$ be a Rademacher sequence in $L_{s}(A)$, where $A \subset B$, and let $R$ be the closed linear span of $\left(r_{n}\right)$ in $L_{s}(A)$. Then, for $\left(\lambda_{n}\right) \in \ell_{2}$ the sum $\sum_{n} \lambda_{n} r_{n}$ is in 
$L_{s}(A)$, hence in $X_{0}(A) \cap X_{1}(A)$ and $\|f\|_{X_{i}} \leq M\|f\|_{s} \leq M^{\prime}\|f\|_{p}$, by Khintchine's inequality. Actually the restriction of the norm of the spaces $X_{0}, X_{1}, L_{p}$ and $L_{s}$ to $R$ is equivalent to the norm of $\left(\lambda_{n}\right)$ in $\ell_{2}$.

Hence for $f \in R$ we may take $F(z)=f$ for all $z \in \mathbb{S}$ since $\|F\|_{\mathcal{F}} \leq M^{\prime}\|f\|_{p}$, and so $\Omega(f)=F^{\prime}\left(\frac{1}{2}\right)=0$. As $\Phi \approx c \Omega$ we see that $\Phi$ is bounded on $R$.

Now suppose $p \leq 1$ and let $\Phi$ be a centralizer on $L_{p}$. We define $r$ by the identity $p^{-1}=r^{-1}+2^{-1}$. Then there is a centralizer $\Psi$ on $L_{2}$ and a constant $M$ such that

$$
\|\Phi(g f)-g \Psi(f)\|_{p} \leq M\|g\|_{r}\|f\|_{2} \quad\left(g \in L_{r}, f \in L_{2}\right)
$$

(see [6, Theorem 8.1] for the case $p=1$ and [2, Corollary 3] for $p<1$ ). We know from the first part of the proof that there is a set $B$ with measure arbitrarily close to 1 such that if $A \subset B$ and $R$ is a subspace of $L_{2}(A)$ spanned by a Rademacher sequence in $A$, then $\Psi$ is bounded on $R:\|\Psi(f)\|_{2} \leq M^{\prime}\|f\|_{2}$ for $f \in R$. Now taking $g=1$ and $f \in R$ we have

$$
\|\Phi(f)-\Psi(f)\|_{p} \leq M\|1\|_{r}\|f\|_{2} \leq M^{\prime \prime}\|f\|_{p},
$$

and so $\Phi$ is also bounded on $R$.

A centralizer $\Phi$ on $L_{p}$ is said to be symmetric if there is a constant $S$ such that

$$
\|\Phi(f \circ \sigma)-\Phi(f) \circ \sigma\|_{p} \leq S\|f\|_{p}
$$

for every $f \in L_{p}$ and every measure preserving Borel automorphism $\sigma$ of $\mathbb{I}$.

The decreasing rearrangement of a real-valued $f \in L_{0}$ is defined by the formula

$$
f^{*}(t)=\inf _{\lambda(B)=t} \sup _{s \in A \backslash B} f(s) \quad(0 \leq t \leq 1)
$$

where $B$ runs over the Borel subsets of $\mathbb{I}$. That is, $f^{*}$ is the only decreasing, right-continuous function having the same distribution as $f$. It is a basic fact from measure theory that for each $f \in L_{0}$, there is a measure preserving Borel automorphism $\sigma$ of $\mathbb{I}$ (depending on $f$ ) such that $f^{*}=f \circ \sigma$ (almost everywhere), and so $f^{*}$ is a true rearrangement of $f$; see [11, Lemma 2].

Note that if $\Phi$ is a symmetric centralizer on $L_{p}$ and $f^{*}=f \circ \sigma$, then $\| \Phi(f)-$ $\left(\Phi\left(f^{*}\right)\right) \circ \sigma^{-1}\left\|_{p} \leq S\right\| f \|_{p}$ and so the map $\Phi_{s}(f)=\left(\Phi\left(f^{*}\right)\right) \circ \sigma^{-1}$ is a symmetric centralizer equivalent to $\Phi$ with the additional property that the distribution of $\Phi_{s}(f)$ depends only on the distribution of $f$.

We emphasize that, in general, centralizers take values in $L_{0}$. For symmetric centralizers we have, however, the following.

Lemma 1. Suppose $0<p<r<\infty$ and let $\Phi$ be a symmetric centralizer on $L_{p}$. If $f \in L_{r}$, then $\Phi f \in L_{p}$.

Proof. It suffices to prove the lemma for real spaces. Let $\Phi_{r}: L_{r} \rightarrow L_{0}$ be the restriction of $\Phi$ to $L_{r}$. This is a centralizer from $L_{r}$ to $L_{p}$, so by the main result in [2] $\Phi_{r}$ must be trivial and there is $\phi \in L_{0}$ and a constant $M$ such that

$$
\left\|\Phi_{r}(f)-\phi f\right\|_{p} \leq M\|f\|_{r} \quad\left(f \in L_{r}\right) .
$$

We claim that $\phi \in L_{s}$, where $s^{-1}+r^{-1}=p^{-1}$. By the Hölder inequality this implies that $\phi f \in L_{p}$, and the same occurs for $\Phi(f)=\Phi_{r}(f)$. To see this, observe that since $f \mapsto \phi f$ is equivalent to $\Phi_{r}$ it is a symmetric centralizer from $L_{r}$ to $L_{p}$, and so there is a constant $S$ such that

$$
\|(\phi \circ \sigma)(f \circ \sigma)-\phi(f \circ \sigma)\|_{p} \leq S\|f\|_{r} \quad\left(f \in L_{r}\right)
$$


whenever $\sigma$ is a measure preserving automorphism of the unit interval. Now, since for every $g \in L_{s}$ one has $\|g\|_{s}=\sup _{\|f\|_{r} \leq 1}\|g f\|_{p}$, we see that $\|\phi \circ \sigma-\phi\|_{s} \leq M^{\prime}$ for some $M^{\prime}$ independent on $\sigma$. By symmetry one also has $\left\|\phi^{*} \circ \sigma-\phi^{*}\right\|_{s} \leq M^{\prime}$, where $\phi^{*}$ is the decreasing arrangement of $\phi$ and $\sigma$ is as before. In particular $\left\|\phi^{*} \circ \sigma-\phi^{*}\right\|_{s}$ is finite when $\sigma(t)=1-t$. Let $m=\phi^{*}\left(\frac{1}{2}\right)$ be the median of $\phi$. Now, since $\phi^{*}$ is decreasing, $\phi^{*} \circ \sigma$ is increasing and both agree with $m$ at $t=\frac{1}{2}$, we see that

$$
\|\phi-m 1\|_{s}=\left\|\phi^{*}-m 1\right\|_{s} \leq\left\|\phi^{*} \circ \sigma-\phi^{*}\right\|_{s}
$$

is finite and $\phi \in L_{s}$.

We are now ready to prove the following.

Proposition 2. Let $0<p<q<2$. There is a subspace $U$ of $L_{p}$ isomorphic to $\ell_{q}$ where the restriction of any symmetric centralizer is bounded.

Proof. It suffices to prove the result for real spaces. Moreover, we may and do assume that the distribution of $\Phi(f)$ depends only on that of $f$.

We proceed as in [12, Proof of Proposition 4.1]. For fixed $q \in(p, 2)$ we consider a $q$-stable random variable $\vartheta \in L_{p}$ and a sequence of independent copies $\left(\vartheta_{n}\right)$. We recall that a random variable is said to be $q$-stable if its characteristic function $(=$ Fourier transform) is $e^{-|t|^{q} / q}$. We refer the reader to [1, Chapter 6, Section 4] for basic information on stable variables. Here we use the following facts:

- If $\vartheta$ is $q$-stable, then $\mathbb{E}\left[|\vartheta|^{r}\right]<\infty$ for $p<r<q$.

- If $\left(\vartheta_{n}\right)$ is a sequence on independent copies of a $q$-stable random variable $\vartheta$ and $\left(\lambda_{n}\right)$ is a sequence normalized in $\ell_{q}$, then $\sum_{n} \lambda_{n} \vartheta_{n}$ has the same distribution as $\vartheta$.

Therefore the map $\left(\lambda_{n}\right) \in \ell_{q} \mapsto \sum_{n} \lambda_{n} \vartheta_{n} \in L_{p}$ is well defined and it is an isometric embedding whose image we denote by $U$. Moreover, by the lemma, $\Phi(\vartheta)$ belongs to $L_{p}$, and then for $\left(\lambda_{n}\right)$ normalized $\ell_{q}$ we have $\left\|\Phi\left(\sum \lambda_{n} \vartheta_{n}\right)\right\|_{p}=\|\Phi(\vartheta)\|_{p}$, and so $\Phi$ is bounded on $U$.

Problem. Is there a strictly singular sequence $0 \rightarrow L_{p} \rightarrow Z \rightarrow L_{p} \rightarrow 0$ for $0<p<2$ ? (See [3, Theorem 2(c)] for the case $2 \leq p<\infty$.)

\section{ACKNOWLEDGEMENTS}

The author thanks Paco Hernández for his help with Köthe spaces. He is grateful to the referee for some remarks that improved the paper.

\section{REFERENCES}

[1] Fernando Albiac and Nigel J. Kalton, Topics in Banach space theory, Graduate Texts in Mathematics, vol. 233, Springer, New York, 2006. MR2192298 (2006h:46005)

[2] F. Cabello Sánchez, Nonlinear centralizers in homology, to appear in Math. Ann. DOI 10.1007/S00208-013-0942-1.

[3] Félix Cabello Sánchez, Jesús M. F. Castillo, and Jesús Suárez, On strictly singular nonlinear centralizers, Nonlinear Anal. 75 (2012), no. 7, 3313-3321, DOI 10.1016/j.na.2011.12.022. MR.2891170

[4] W. B. Johnson, B. Maurey, G. Schechtman, and L. Tzafriri, Symmetric structures in Banach spaces, Mem. Amer. Math. Soc. 19 (1979), no. 217, v+298. MR527010 (82j:46025)

[5] N. J. Kalton, Applications of an inequality for $H_{1}$-functions, Texas Functional Analysis Seminar 1985-1986 (Austin, TX, 1985-1986), Longhorn Notes, Univ. Texas, Austin, TX, 1986, pp. 41-51. MR.1017041 (90k:46033) 
[6] Nigel J. Kalton, Nonlinear commutators in interpolation theory, Mem. Amer. Math. Soc. 73 (1988), no. 385, iv+85. MR938889 (89h:47104)

[7] N. J. Kalton, Differentials of complex interpolation processes for Köthe function spaces, Trans. Amer. Math. Soc. 333 (1992), no. 2, 479-529, DOI 10.2307/2154047. MR1081938 (92m:46111)

[8] Nigel Kalton and Stephen Montgomery-Smith, Interpolation of Banach spaces, Handbook of the geometry of Banach spaces, Vol. 2, North-Holland, Amsterdam, 2003, pp. 1131-1175, DOI 10.1016/S1874-5849(03)80033-5. MR1999193(2006c:46061)

[9] N. J. Kalton and N. T. Peck, Twisted sums of sequence spaces and the three space problem, Trans. Amer. Math. Soc. 255 (1979), 1-30, DOI 10.2307/1998164. MR542869 (82g:46021)

[10] Richard Rochberg and Guido Weiss, Derivatives of analytic families of Banach spaces, Ann. of Math. (2) 118 (1983), no. 2, 315-347, DOI 10.2307/2007031. MR717826 (86a:46099)

[11] John V. Ryff, Orbits of $L^{1}$-functions under doubly stochastic transformations, Trans. Amer. Math. Soc. 117 (1965), 92-100. MR0209866 (35 \#762)

[12] J. Suárez, The Kalton centralizer on $L_{p}$ is not strictly singular. Proc. Amer. Math. Soc. 141 (2013), no. 10, 3447-3451. MR 3080167

Departamento de Matemáticas, Universidad de Extremadura, 06071-Badajoz, Spain

E-mail address: fcabello@unex.es 